\title{
Great in practice, not in theory: An empirical examination of covered call writing
}

\author{
Michael L. Mclntyre*, and David Jackson \\ ${ }^{*}$ Eric Sprott School of Business, Carleton University, 1125 Colonel By Drive, Ottawa, \\ Ontario, Canada K1S 5B6. \\ Tel: + 1613520 2600, Fax: + 1613520 4427, E-mail: mmcintyr@sprott.carleton.ca \\ Received (in revised form): 4th November, 2006
}

\begin{abstract}
Michael L. McIntyre is Assistant Professor in the Eric Sprott School of Business at Carleton University. Prior to entering academia, he worked as a Chartered Accountant and in corporate banking with a major Canadian bank.

David Jackson is Assistant Professor in the Eric Sprott School of Business at Carleton University. Prior to entering academia, he worked as an engineer, primarily in instrumentation and developing special purpose computer-based data acquisition and control systems.
\end{abstract}

\section{Practical applications}

Covered call writing is a common investment approach among individual investors and money managers. Although these investors often argue for the practice on the grounds that income from covered call writing can enhance overall returns during periods when stock prices are languishing, it had not been previously demonstrated that the practice is empirically supported. The practical application of this paper is that it provides an empirical grounding that investors can use when deciding on the merits of a covered call writing investment strategy.

\begin{abstract}
We examine the empirical performance of an investment strategy that uses covered call writing to enhance the income from long positions in 27 stocks that are included in the FT-SE 100 Index. Using data for the period January 1994-December 1999 we show that, contrary to theory, in most instances covered call positions generate returns that exceed returns generated by buy-and-hold strategies. Journal of Derivatives \& Hedge Funds (2007) 13, 66-79. doi:10.1057/palgrave.jdhf.1850063
\end{abstract}

Keywords: portfolio strategy; derivatives

\section{INTRODUCTION}

There are various mutual funds in existence ${ }^{1}$ that claim to enhance returns to unit holders through writing call options on investments held in the funds. This strategy is called 'covered call writing' when the number of options written on a stock does not exceed the number of shares of that stock held in the portfolio. Proponents of the covered call strategy assert that the portfolio benefits from a price increase ${ }^{2}$ and the call premium under conditions when the stock is called out, and benefits from the call premium if the stock is not called out. In the latter instance,
Journal of Derivatives \& Hedge Funds, Vol. 13 No. 1, 2007, pp. 66-79 (C) 2007 Palgrave Macmillan Ltd $1753-9641 \$ 30.00$ 
income from writing the call mitigates the drag on returns due to stagnancy or decline in the price of a portfolio member.

While these arguments are superficially appealing, closer inspection suggests that covered call writing may militate against enhancing portfolio returns. To see this, think of a covered call position as a portfolio comprised of a long position in the underlying financed in part by a short position in an option on the same underlying. Using $a$ and $b$ to denote positive portfolio weights where $a$ is greater than 1 , and $a-b=1$, the initial condition is the following:

$$
\underbrace{a S_{t}-b C_{t}}_{\text {Covered Call }}=\underbrace{S_{t}}_{\text {Buy and Hold }}
$$

where $S_{t}$ is the price of the underlying asset and $C_{t}$ is the price of the call. In a Black and Scholes ${ }^{3,4}$ world, the option can be replicated using a long position in $\delta_{t}$ units of the underlying asset, where $0<\delta_{t}<1$, and a short position in a riskless bond so that:

$$
C_{t}=\delta_{t} S_{t}-B_{t}
$$

Substituting the expression in (2) into (1) gives:

$$
a S_{t}-b\left(\delta_{t} S_{t}-B_{t}\right)=\left(a-\delta_{t} b\right) S_{t}+b B_{t}
$$

The expression in (3) indicates that entering a covered call position amounts to increasing one's position in a riskless bond in substitution for the underlying. In most cases one would think of the expected return on the underlying exceeding the return on the riskless bond, so writing covered calls typically would reduce expected returns.

\section{MONTE CARLO SIMULATION}

To illustrate the case in which the expected return on the underlying exceeds the return on the riskless bond $\left(E\left[r_{i}\right]>r_{f}\right)$, we constructed a 50,000 path Monte Carlo simulation using five economic specifications. The economies are the Black-Scholes ${ }^{3}$ economy (BS), the $\mathrm{Cox}^{5}$ constant elasticity of variance economy (CEV), the Geske ${ }^{6}$ compound option-pricing model (LEV), the Merton ${ }^{7}$ jump-diffusion economy (JD), and the Heston ${ }^{8}$ stochastic volatility economy (HES). Details of each of these economies are presented in the Appendix. In each of these economies, we adopted covered call strategies using options with, respectively, 30, 60, and 90 days to maturity.

We chose model parameters, shown in Table 1 , so that in each economy the price of a 30-day, at-the-money European style call option approximated the Black-Scholes price with volatility set at 15 per cent. In Table 1 , the percentages associated with strike prices $K_{1}$ and $K_{2}$ denote the degree of moneyness ${ }^{9}$ at which the near-the-money and out-of-the-money options were written in the simulations. Note that the comparison of covered call strategies to the buy-and-hold strategies in this paper are all 'within model' and not 'across models' so it is not necessary to establish a correspondence between models that goes beyond the qualitative similarity implied by the above approach.

As set out in Figure 1, the pattern of covered call writing we adopted in the simulations matches the pattern that is feasible using transactions data. Although Monte Carlo simulations permit one to assume the existence of options with any desired maturities, we elected to render our simulations consistent with the coverage pattern that can be achieved with transactions data. For the stocks included in this study (see Table 4 below), option prices were quoted for options with 90-day increments in time to maturity. This permitted the coverage pattern for written calls that is shown in Figure 1. For example, in the 30-day written call protocol, 
Table 1: Parameter values

\begin{tabular}{|c|c|c|c|c|c|}
\hline & \multicolumn{5}{|l|}{ Economies } \\
\hline & Black-Scholes & Leveraged & $C E V$ & Heston & Jump/Diffusion \\
\hline$S_{0}$ & 100 & 100 & 100 & 100 & 100 \\
\hline$V$ & - & 150 & - & - & - \\
\hline$M(t, T)$ & - & 50 & - & - & - \\
\hline$T-t$ & - & 10 years & - & - & - \\
\hline$K_{1}$ & $102 \%$ & $102 \%$ & $102 \%$ & $102 \%$ & $102 \%$ \\
\hline$K_{2}$ & $105 \%$ & $105 \%$ & $105 \%$ & $105 \%$ & $105 \%$ \\
\hline$\mu$ & 0.12 & 0.095 & 0.12 & 0.12 & 0.12 \\
\hline$R$ & 0.05 & 0.05 & 0.05 & 0.05 & 0.05 \\
\hline$Q$ & 0 & 0 & 0 & 0 & 0 \\
\hline$\alpha$ & - & - & $4 / 3$ & - & 0.13 \\
\hline$\sqrt{v}$ & 0.15 & 0.10 & $\sqrt{0.5}$ & 0.15 & $\sqrt{0.16}$ \\
\hline$\theta$ & - & - & - & 0.15 & - \\
\hline$\kappa$ & - & - & - & 20.0 & 0 \\
\hline$\sigma$ & - & - & - & 0.10 & - \\
\hline$\rho$ & - & - & - & -0.5 & - \\
\hline$\lambda$ & - & - & - & - & 2 jumps/year \\
\hline$\beta$ & - & - & - & - & $\sqrt{0.006}$ \\
\hline
\end{tabular}

Maturity of
Written Calls
90 days
60 days
30 days
Denotes period during which the portfolio is short the written call

Figure 1: Coverage pattern of written calls

we left the position uncovered (ie no call was written) for 60 days until the shortest maturity call that was quoted on the market had 30 days left to maturity. At this point, we wrote a 30-day call at the quoted market price. In the next 90-day cycle, we followed the same protocol. Similarly, the 60-day options left the covered call position uncovered one-third of the time.
The simulation results are set out in Table 2 . In addition to including strategies involving 30, 60, and 90-day options, respectively, we tracked covered call positions for two levels of strike price. In the first case, we assumed that the written option possessed a strike price, $K_{1}$, 2 per cent out of the money. In the second case we assumed a strike price, $K_{2}, 5$ per cent out of 
Table 2: Summary of simulation results

\begin{tabular}{|c|c|c|c|c|c|c|}
\hline & \multicolumn{2}{|l|}{30 day } & \multicolumn{2}{|l|}{60 day } & \multicolumn{2}{|l|}{90 day } \\
\hline & $K_{1}(\%)$ & $K_{2}(\%)$ & $K_{1}(\%)$ & $K_{2}(\%)$ & $K_{1}(\%)$ & $K_{2}(\%)$ \\
\hline \multicolumn{7}{|c|}{ Panel A: Covered call returns as a percent of buy-and-hold returns } \\
\hline BS & 93.7 & 98.7 & 84.8 & 93.9 & 75.5 & 87.3 \\
\hline $\mathrm{CEV}$ & 93.7 & 98.9 & 84.4 & 94.5 & 74.5 & 87.9 \\
\hline LEV & 94.1 & 98.9 & 85.5 & 94.6 & 76.6 & 88.5 \\
\hline JD & 94.2 & 99.3 & 85.6 & 94.8 & 76.4 & 88.2 \\
\hline HES & 93.5 & 98.8 & 84.0 & 93.9 & 74.0 & 86.8 \\
\hline \multicolumn{7}{|c|}{ Panel B: Covered call volatility as a percent of buy-and-hold volatility } \\
\hline BS & 90.2 & 97.5 & 75.8 & 88.7 & 55.6 & 75.6 \\
\hline $\mathrm{CEV}$ & 90.7 & 98.4 & 75.9 & 90.7 & 55.4 & 78.3 \\
\hline LEV & 91.0 & 98.0 & 76.8 & 90.4 & 56.9 & 78.2 \\
\hline JD & 90.7 & 96.3 & 76.7 & 87.6 & 58.1 & 75.1 \\
\hline HES & 90.6 & 97.7 & 76.9 & 90.0 & 57.6 & 77.8 \\
\hline
\end{tabular}

Table 3: Percentage of paths with covered call returns in excess of buy-and-hold returns

\begin{tabular}{lll}
\hline & \multicolumn{2}{l}{90 day } \\
\cline { 2 - 3 } & $K_{1}(\%)$ & $K_{2}(\%)$ \\
\hline BS & 21 & 38 \\
CEV & 17 & 39 \\
LEV & 22 & 41 \\
JD & 27 & 41 \\
HES & 21 & 39 \\
\hline
\end{tabular}

the money. The results indicate that in all cases, the mean returns from the covered call positions were less than for the buy-and-hold positions. Also, in all cases, the volatilities of returns from the covered call positions were less than for the buy-and-hold positions.
Table 3 presents data on the frequency with which returns from the covered call positions exceeded returns from the buy-and-hold positions. Notwithstanding the results for the mean shown in Table 2, Table 3 shows that in qualitative terms, the 'losing' covered call strategy outperforms the buy-and-hold strategy relatively frequently. Arguably, participants in a market which exhibits this characteristic could develop a favourable perception of the merits of covered call writing even though it is a losing strategy overall.

\section{DATA}

We obtained the data used in this paper from the LIFFE Euro-Out Products tick data disc. We randomly selected 30 companies from among the constituents of the FT-SE 100 Index as on 31st December, 1999. The LIFFE Euro-Out 
Products tick data disc includes option price ${ }^{10}$ data for a wide variety of traded options along with contemporaneous prices for the underlying. We deleted one company from the study because it was unclear whether a stock split reported by the company had been properly accounted for in the underlying price time series on the data disc. ${ }^{11}$ We deleted two companies because of insufficient numbers of quoted option prices. Table 4 sets out the names and stock symbols of the companies included in the study.

Table 5 reports the returns, volatility, and the coefficient of correlation between returns on the individual stocks and the FT-SE 100 Index for the sample data. The reported returns are the annualised log-differences of share price at the beginning and end of the timeframe the study covers. The reported standard deviations of returns are the annualised standard deviations of daily returns over the timeframe the study covers. The reported correlations are also based on daily returns observations.

The coefficients of correlation for all companies were positive. Although a more sophisticated estimation of time varying coefficients of correlation, and a broader definition of the market portfolio would be desirable, the historical coefficients are suggestive of the possibility that each of the stocks is a positive beta stock in a capital asset pricing model sense. This being the case, the return expected on each stock exceeds the return on a riskless bond. Consequently, as shown in (3), our ex ante expectation is that mean returns for the buy-and-hold strategies should exceed mean returns for the covered call strategies. ${ }^{12}$

Table 4: Companies included in the sample and related stock symbols

\begin{tabular}{llll}
\hline Stock & Company name & Stock & Company name \\
symbol & & symbol & \\
\hline ALD & Allied Domecq PLC & MS & Marks and Spencer PLC \\
BBL & Barclays PLC & PO & Peninsular and Oriental Steam \\
& & & Navigation Company PLC \\
BOT & Boots Company PLC & RTZ & Rio Tinto PLC \\
BP & British Petroleum Company PLC & RUT & Reuters Holdings PLC \\
BSS & Bass PLC & RYL & Royal and Sun Alliance \\
& & & Insurance Group PLC \\
CAD & Cadbury Schweppes PLC & SAN & Sainsbury (J) PLC \\
CIR & Blue Circle Industries PLC & STL & Corus Group PLC (formerly British Steel PLC) \\
CUA & Commercial General Union PLC & TAR & Tarmac PLC \\
CW & Cable and Wireless PLC & TCO & Tesco PLC \\
DIX & Dixon's Group PLC & TW & Thames Water PLC \\
GXO & Glaxo Wellcome PLC & ULV & Unilever PLC \\
HSB & HSBC Holdings PLC & VOD & Vodafone Air Touch PLC \\
ICI & Imperial Chemical Industries PLC & ZCA & Zeneca Group PLC \\
LMO & LASMO PLC & & \\
\hline
\end{tabular}


Table 5: Realised returns, volatilities, and correlation with the FTSE 100 Index

\begin{tabular}{llllllll}
\hline Company & $\begin{array}{l}\text { Annual } \\
\text { returns }(\%)\end{array}$ & $\begin{array}{l}\text { Volatility } \\
(\%)\end{array}$ & $\begin{array}{l}\text { Correl. } \\
\text { coeff. }\end{array}$ & Company & $\begin{array}{l}\text { Annual } \\
\text { returns }(\%)\end{array}$ & $\begin{array}{l}\text { Volatility } \\
(\%)\end{array}$ & $\begin{array}{l}\text { Correl. } \\
\text { coeff. }\end{array}$ \\
\hline ALD & -3.43 & 24.09 & 0.39 & MS & 0.35 & 34.69 & 0.41 \\
BBL & 17.11 & 29.35 & 0.65 & PO & -7.07 & 26.51 & 0.43 \\
BOT & 0.35 & 23.19 & 0.38 & RTZ & 7.89 & 25.02 & 0.44 \\
BP & 20.92 & 23.65 & 0.29 & RUT & 9.51 & 25.64 & 0.44 \\
BSS & -1.20 & 44.85 & 0.37 & RYL & 14.63 & 33.41 & 0.58 \\
CAD & 5.82 & 26.07 & 0.37 & SAN & 5.42 & 32.95 & 0.37 \\
CIR & 5.60 & 23.60 & 0.33 & STL & -4.18 & 26.06 & 0.28 \\
CUA & 0.86 & 31.45 & 0.58 & TAR & 3.94 & 33.55 & 0.27 \\
CW & 7.19 & 29.80 & 0.54 & TCO & -5.12 & 32.56 & 0.39 \\
DIX & 11.30 & 33.00 & 0.28 & TW & 15.92 & 26.57 & 0.30 \\
GXO & 27.26 & 32.48 & 0.60 & ULV & 4.88 & 22.15 & 0.53 \\
HSB & 14.86 & 28.03 & 0.69 & VOD & 6.94 & 24.31 & 0.52 \\
ICI & 16.13 & 31.86 & 0.36 & ZCA & 33.43 & 33.11 & 0.50 \\
LMO & -3.03 & 29.43 & 0.24 & & & & \\
\hline
\end{tabular}

For each stock in the study, we tracked a buy-and-hold position concurrent with a covered call position. In each case, we did so for three different maturities of written option with the coverage patterns following those described in Figure 1. For each maturity, we tracked a covered call strategy for two levels of moneyness. For the first level, we used options with a strike price out of the money, but nearest to the at-themoney level. For the second level, we used the next available out-of-the-money strike price. We wrote options in a quantity equal to the holdings in the underlying at the beginning of each of the coverage periods shown in Figure 1 and used the option premiums from the written calls to increase the number of units of the underlying in the covered call position. If the stock was called out, we deleted units from our position equal in amount to the number of written calls in the call-out, and used the proceeds from the call-out (strike price times the number of written calls in the call-out) to acquire as many units of the underlying as possible at the price of the underlying at the time of the call-out. At the end of the study period, we calculated the annualised returns for the covered call position and the buy-and-hold position, respectively. We did not take account of transaction costs involved in either investment strategy because the goal of this paper is to examine the comparative properties of the underlying and options on the underlying rather than to determine relative economic merits.

\section{RESULTS AND DISCUSSION}

Table 6 summarises our results. In all but one of the scenarios, covered call writing strategies produced better returns more frequently than the buy-and-hold strategy. We consider the 
Table 6: Annualised returns from covered call writing compared to the buy-and-hold strategy

\begin{tabular}{|c|c|c|c|c|c|c|c|}
\hline & \multicolumn{7}{|c|}{ Covered call } \\
\hline & \multicolumn{2}{|l|}{30 Day } & \multicolumn{2}{|l|}{60 Day } & \multicolumn{2}{|l|}{90 Day } & \multirow[t]{2}{*}{ Buy and Holo } \\
\hline & $A(\%)$ & $B(\%)$ & $A(\%)$ & $B(\%)$ & $A(\%)$ & $B(\%)$ & \\
\hline \multicolumn{8}{|l|}{ Panel $A$} \\
\hline ALD & -4.20 & -3.12 & 0.18 & -1.34 & 1.13 & -0.47 & -3.43 \\
\hline $\mathrm{BBL}$ & 13.99 & 15.84 & 16.55 & 18.94 & 7.78 & 12.34 & 17.11 \\
\hline BOT & 2.45 & 2.06 & 4.74 & 3.00 & 2.54 & 1.71 & 0.35 \\
\hline $\mathrm{BP}$ & 23.14 & 23.31 & 21.90 & 22.71 & 20.95 & 22.63 & 20.92 \\
\hline BSS & -10.51 & -10.88 & -1.85 & -3.19 & -4.96 & -3.61 & -1.20 \\
\hline CAD & 3.25 & 6.47 & 8.96 & 8.30 & 7.94 & 8.12 & 5.82 \\
\hline CIR & 7.47 & 7.79 & 8.72 & 9.27 & 8.70 & 9.72 & 5.60 \\
\hline CUA & 7.21 & 3.01 & 6.29 & 4.93 & 7.84 & 5.34 & 0.86 \\
\hline $\mathrm{CW}$ & 5.11 & 8.36 & 8.36 & 9.25 & 8.60 & 8.87 & 7.19 \\
\hline DIX & 6.37 & 8.20 & 11.36 & 13.12 & 7.63 & 8.95 & 11.30 \\
\hline GXO & 25.46 & 27.17 & 22.30 & 25.03 & 11.78 & 16.03 & 27.26 \\
\hline HSB & 13.12 & 15.88 & 13.65 & 15.88 & 6.91 & 11.46 & 14.86 \\
\hline ICI & 8.29 & 11.35 & 12.76 & 15.34 & 6.87 & 8.66 & 16.13 \\
\hline LMO & -4.81 & -1.17 & -0.74 & -0.41 & -0.39 & -1.51 & -3.03 \\
\hline MS & 4.79 & 4.30 & 8.34 & 6.17 & 8.24 & 5.85 & 0.35 \\
\hline $\mathrm{PO}$ & -7.12 & -6.99 & -5.37 & -6.21 & -4.61 & -6.51 & -7.07 \\
\hline RTZ & 8.53 & 10.81 & 11.79 & 12.01 & 8.03 & 10.40 & 7.89 \\
\hline RUT & 6.89 & 8.67 & 6.57 & 7.72 & 6.01 & 8.33 & 9.51 \\
\hline RYL & 18.58 & 16.53 & 21.91 & 20.78 & 18.06 & 18.32 & 14.63 \\
\hline SAN & 3.67 & 7.25 & 7.81 & 8.92 & 4.16 & 5.97 & 5.42 \\
\hline STL & -3.49 & -3.83 & -1.05 & -0.88 & -0.11 & -2.25 & -4.18 \\
\hline TAR & 1.06 & 2.50 & 5.41 & 6.81 & 8.97 & 8.14 & 3.94 \\
\hline TCO & 2.48 & -1.28 & 0.34 & -0.28 & 0.45 & -0.79 & -5.12 \\
\hline TW & 19.72 & 18.31 & 18.38 & 18.90 & 19.38 & 20.40 & 15.92 \\
\hline ULV & 2.51 & 4.28 & 3.83 & 3.70 & 4.04 & 4.55 & 4.88 \\
\hline VOD & 10.75 & 8.72 & 7.20 & 8.77 & 6.53 & 7.71 & 6.94 \\
\hline ZCA & 30.39 & 33.91 & 23.06 & 28.18 & 22.99 & 25.10 & 33.43 \\
\hline \multicolumn{8}{|l|}{ Panel B } \\
\hline VWP & 10.93 & 11.90 & 11.82 & 12.78 & 9.26 & 10.42 & 11.50 \\
\hline EWP & 10.35 & 11.45 & 10.83 & 11.90 & 8.75 & 9.81 & 10.95 \\
\hline \multicolumn{8}{|l|}{ Panel C } \\
\hline Winners & 11 & 19 & 19 & 21 & 16 & 18 & \\
\hline
\end{tabular}

A denotes the nearest the money strategy, and B denotes the next-nearest the money strategy. 
statistical significance of these outcomes using the 'sign test for matched pairs' and the 'Wilcoxon signed rank test'. These statistical methods and the expression of the null hypothesis in the context of them are discussed below. $^{13,14}$

The sign test for matched pairs applies in situations in which there is either a failure or a success arising from repeated independent trials and there is a constant probability, $p$, of success. The test permits inference about the hypothesised probability, $p$. In the current context, the trials are the attempts to earn superior returns through the buy-and-hold strategy versus the covered call strategy. One would hypothesise that, if each strategy is equally effective, there would be an equal chance that one strategy would outperform the other at each comparison point. Thus, in the context of the sign test for matched pairs, the null hypothesis is the following:

$H_{0}$ : The probability that buy and hold outperforms covered call writing for each attempt is $\frac{1}{2}$.

The assumptions underlying the test are the following:

1 the outcomes can be classified as a success or a failure

2 the probability of a success remains constant from trial to trial and

3 the trials are independent.

There were no ties in the tests. The null hypothesis is that the probability of a success or failure is equal for each comparison of investment protocols. The hypothesised probability is, therefore, constant.
For larger samples $(N>20)$, the test statistic $z$ for the sign test for matched pairs can be determined in relation to either $P_{M}$, where $P_{M}$ is the number of occurrences of the more frequent outcome divided by the number of trials $N$, or in relation to $P_{L}$, where $P_{L}$ is the number of occurrences of the less frequent outcome divided by the number of trials $N$. There are therefore two possible $z$-scores, $Z_{M}$ determined using $P_{M}$, and $Z_{L}$ determined using $P_{L}$. It is easy to show that for hypothesised $p=\frac{1}{2}, z_{M}=-z_{L}$ so, from an inference point of view, and consistent with the two-sided hypothesis stated above, it makes no difference whether one bases the statistical test on $P_{M}$ or $P_{L}$. The sole effect of basing the $z$-score calculation on $P_{M}$ versus $P_{L}$ is that in the first instance the correction for continuity in the $z$-score calculation is $-\frac{1}{2} \mathrm{~N}$, whereas in the second instance it is $+\frac{1}{2} N$. Expressions for both possible $z$-scores are set out below:

$$
\begin{aligned}
z_{M} & =\frac{\left(P_{M}-p\right)-1 / 2 N}{\sqrt{p q / N}} \\
z_{L} & =\frac{\left(P_{L}-p\right)+1 / 2 N}{\sqrt{p q / N}}
\end{aligned}
$$

In equations (4), $z_{M}$ and $z_{L}$ are the usual $z$-score associated with the unit normal distribution. $p$ is the hypothesised probability, and $q$ is $1-p$. The probability associated with the test statistic $z_{M}\left(z_{L}\right)$ is interpreted as the probability that $P_{M}$ or greater $\left(P_{L}\right.$ or less) was obtained by chance given a true probability $p$ equal to its hypothesised value.

The Wilcoxon signed rank test is also a test for population differences in matched pairs. It takes account not only of the number of occurrences of a particular outcome but also takes account of the rank of the absolute size of each difference. This test, therefore, uses more information from 
a particular test than the sign test for matched pairs. As in the preceding case, comparison of individual returns performance is between the buy-and-hold strategy and the covered call strategy.

The assumptions underlying the Wilcoxon signed rank test are the following:

1 Define $Z_{i}$ such that $Z_{i} \equiv r_{C C}-r_{B \& H}$, where $r_{B \& H}$ denotes returns from the buy-and-hold strategy and $r_{C C}$ denotes returns from the covered call strategy, both in respect of stock $i$. The assumed underlying model is:

$$
Z_{i}=\theta+e_{i}
$$

In (5), $\theta$ is the hypothesised differential in investment strategies and the $e_{i}$ are unobservable random variables.

2 The unobservable random variables $e_{i}$ are mutually independent.

3 Each $e_{i}$ comes from a continuous population that is symmetric about zero. The populations do not need to be identical.

The null hypothesis in the context of the Wilcoxon signed rank test is:

$$
H_{0}: \theta=0
$$

To calculate the test statistic $T, \operatorname{rank}^{15}$ the absolute values of $Z_{i}$ so that there is a rank value $R_{i}$ corresponding to each $Z_{i}$. Define the indicator variable $\psi_{i}$ to equal one if $Z_{i}$ is positive and zero otherwise. If $\sum \psi_{i}<\sum\left(1-\psi_{i}\right)$, the test statistic $T$ is $\sum R_{i} \psi_{i}$. Otherwise, it is $\sum R_{i}\left(1-\psi_{i}\right)$

For large samples, $T$ is normally distributed with:

$$
E[T]=\frac{N(N+1)}{4}
$$

and

$$
\operatorname{VAR}[T]=\frac{N(N+1)(2 N+1)}{24}
$$

The test score is:

$$
z=\frac{T-E[T]}{\sqrt{\operatorname{VAR}[T]}}
$$

In (7), $T$ is the test statistic calculated as described above. In (8), $z$ is the usual $z$-score associated with the unit normal distribution. If $z$ is less than (greater than) zero, the probability associated with the test statistic $z$ is interpreted as the probability of obtaining the test statistic or less (or more) given that the populations of returns differentials are symmetrical with identical median.

As with the sign test for matched pairs, inferences are identical whether the test statistic $z$ is determined from $T$ calculated using the less frequently occurring outcome, as above, or if $z$ is determined from $T$ calculated using the more frequently occurring outcome. As a consequence, and consistent with the two-sided nature of the hypothesis stated above, one need not make an ex ante selection of a 'preferred' or 'winning' investment protocol to calculate the test statistic. Define $T_{L}$ equal to $T$ calculated using the less frequently occurring outcome, $T_{M}$ equal to $T$ calculated using the more frequently occurring outcome, and corresponding $z$-scores $z_{L}$ and $z_{M}$. It can be shown that $z_{L}=-z_{M}$. This being the case, there can be no difference in inference in relation to the two-sided hypothesis stated above resulting from calculating $z_{L}$ rather than $z_{M}$, or vice versa.

Given all of the assumptions set out above, the Wilcoxon signed rank test constitutes a test for location. If the error terms cannot be assumed to be symmetrically distributed, the Wilcoxon signed rank test constitutes a joint test for the median and for symmetry (cf. Gibbons and Chakraborti, ${ }^{16}$ pp. $\left.168-169\right)$. In this 
circumstance, the null hypothesis in the context of the Wilcoxon signed rank test is:

$H_{0}$ : The population of differences in investment protocols is symmetric with median $\theta=0$.

If the joint null hypothesis stated above is rejected, it is not possible to determine whether the failure is attributable to one or both elements of the null hypothesis.

To facilitate discussion, we arbitrarily use the term winner to identify cases where returns from covered call writing exceed returns from the buy-and-hold strategy. The column headings $\mathrm{A}$ and $\mathrm{B}$ in Table 7 denote, respectively, the nearest the money option and the next-nearest the money option. Panel A in Table 7 summarises the results of the sign test for matched pairs. The results for 30-day options next-nearest the money, and 60-day options both nearest and next-nearest the money, and 90-day options next-nearest the money indicate that the covered call writing strategy produced an unusual number of winners. The results for 60-day options nextnearest the money indicate that the probability that the realised number of winners occurred by chance conditional on an hypothesised probability of 50 per cent was just 0.4 per cent. These results suggest that the hypothesis under the sign test for matched pairs is not supported by the data for at least some of the covered call writing protocols.

Panel B of Table 7 summarises the results for the Wilcoxon signed rank test. In each case the test statistic $z$ is negative. The probability associated with the test statistic $z$ is interpreted as the probability of obtaining the test statistic or less given that the populations of returns differentials are symmetrical with identical median. For the most part, the results in Panel B support those obtained in Panel A with the possible exception of the result for 90-day nextnearest the money. The test result under the

Table 7: Results of statistical tests

\begin{tabular}{|c|c|c|c|c|c|c|}
\hline & \multicolumn{2}{|l|}{30 Day } & \multicolumn{2}{|l|}{60 Day } & \multicolumn{2}{|l|}{90 Day } \\
\hline & $A$ & $B$ & $A$ & $B$ & $A$ & $B$ \\
\hline \multicolumn{7}{|c|}{ Panel A: Sign test for matched pairs } \\
\hline Winners & 11 & 19 & 19 & 21 & 16 & 18 \\
\hline Losers & 16 & 8 & 8 & 6 & 11 & 9 \\
\hline Max & 16 & 19 & 19 & 21 & 16 & 18 \\
\hline$z$ & 0.7698 & 1.9245 & 1.9245 & 2.6943 & 0.7698 & 1.5396 \\
\hline$p$-value & 0.221 & 0.027 & 0.027 & 0.004 & 0.221 & 0.062 \\
\hline \multicolumn{7}{|c|}{ Panel B: Wilcoxon signed rank test } \\
\hline$T$ & 168 & 115 & 102 & 58 & 182 & 148 \\
\hline$z$ & -0.5045 & -1.7778 & -2.0902 & -3.1473 & -0.1682 & -0.9850 \\
\hline$p$-value & 0.307 & 0.038 & 0.018 & 0.001 & 0.433 & 0.162 \\
\hline
\end{tabular}


latter test is less extreme than for the former test. The difficulty with the Wilcoxon signed rank test is that its interpretation is unclear if the error terms in the model cannot be assumed symmetrically distributed. The stylised facts of returns distributions suggest that there is the possibility of asymmetrically distributed stock returns. This therefore calls into question whether the hypothesis fails due to asymmetry or due to a median $\theta \neq 0$.

\section{CONCLUSIONS AND FUTURE RESEARCH}

In this paper, we present results of a comparison of two investment protocols. In the first instance, the investment protocol involves a buy-and-hold strategy. In the second instance, it involves writing covered calls on a long position in an underlying stock. We conduct the comparison in synthesised economies to demonstrate the likely outcome if the real economy were consistent with the theoretical models in the synthesised economies. We also conduct the comparison using transactions data. In the latter case, we find a surprising number of cases in which the covered call strategy produces greater returns than the buy-and-hold strategy, with some evidence that this outcome is statistically significant. This result is surprising considering the clear evidence in the synthesised economies that this result does not occur when tested over many repeated trials. The evidence from the synthesised economies indicates, however, that even though the buy-and-hold strategy produces greater returns when an average is taken over many repeated trials, a qualitative assessment of the covered call strategy suggests it performs well relatively frequently.

\section{References and Notes}

1 cf. the PRO-AMS, PRO-AMS US, and Triax CARTS funds, for example.

2 In practice, covered call writing involves writing options that are somewhat out-of-the-money.

3 Black, F. and Scholes, M. (1973) 'The Pricing of Options and Corporate Liabilities', Journal of Political Economy, Vol. 81, pp. 637-654.

4 We recognise that there are various limitations of the Black-Scholes pricing approach, but we submit that the approach is qualitatively appropriate in this instance.

5 Cox, J.C. (1975) 'Notes on Option Pricing 1: Constant Elasticity of Variance Diffusions’, unpublished manuscript, Stanford University.

6 Geske, R. (1979) 'The Valuation of Compound Options', Journal of Financial Economics, Vol. 7(March), pp. 63-81.

7 Merton, R.C. (1976) 'Option Pricing When Underlying Stock Returns are Discontinuous', Journal of Financial Economics, Vol. 3, pp. 125-144.

8 Heston, S.L. (1993) 'A Closed-Form Solution for Options With Stochastic Volatility With Application to Bond And Currency Options', Review of Financial Studies, Vol. 6, pp. 327-343.

9 By moneyness, we mean the extent to which the option is out of the money.

10 Prices are in respect of American style options. Although the options used in the study possessed the early exercise feature, there is no evidence to suggest that early exercise occurred during the life of these options.

11 We also calculated results including this company and our best estimate of appropriate treatment for the apparent stock split. There was no material difference in the conclusions of the study.

12 Since both strategies include a long position in the underlying, we did not explicitly take account of dividends in the cash flows arising from each of the respective positions.

13 Hollander, M. and Wolfe, D.A. (1973) 'Nonparametric Statistical Methods', John Wiley \& Sons, New York.

14 Hollander and Wolfe ${ }^{13}$ present and explain both of the statistical methods used in this paper. The comments on the statistical methods in this section follow the comments and analysis in their Chapters 2 and 3.

15 The ranking is in ascending order so that the smallest absolute value receives a rank of one.

16 Gibbons, J.D. and Chakraborti, S. (1992) 'Nonparametric Statistical Inference, 3rd edn. Marcel Dekker, Inc, New York. 


\section{Appendix 1}

\section{BLACK-SCHOLES}

In this model, the price of the underlying asset $S$ is assumed to follow the stochastic process:

$$
\mathrm{d} S=\mu S \mathrm{~d} t+\sigma S \mathrm{~d} Z
$$

The discrete time version of (A1) is:

$$
S\left(t_{i+1}\right)=S\left(t_{i}\right) \exp [(\mu-v / 2) \Delta t+(\sqrt{v} x \sqrt{\Delta t})]
$$

where $x$ is a random draw from the unit normal distribution.

\section{LEVERAGED MODEL}

The Geske ${ }^{6}$ compound option pricing model is used to model option pricing in the context of a firm with financial leverage. Geske ${ }^{6}$ assumes that firm value, $V$, follows the stochastic process:

$$
\mathrm{d} V=\mu V \mathrm{~d} t+\sqrt{v} V \mathrm{~d} Z
$$

He also assumes that the firm has issued a pure discount bond that gives the holder the right to amount $M$ at maturity $T$. Denote its value at the present time $t$ by $M(t, T)$ and at maturity by $M(T, T)$. The firm is not permitted to issue pari passu or senior ranking claims, nor is it permitted to make distributions or share repurchases prior to $T$. Under this specification, the bondholders can be viewed as 'owning' the firm with the common shareholders having an option to acquire ownership at $T$ upon payment to the bondholders of the amount $M(T, T)$. A call option on a common share is therefore an option on an option. Geske ${ }^{6}$ shows that the value of a common share, $S$, is given by the Black-Scholes call-option pricing formula and develops a pricing expression for the value of a call option on $S$.
The following is the discrete time version of (A3):

$$
V\left(t_{i+1}\right)=V\left(t_{i}\right) \exp [(\mu-v / 2) \Delta t+(\sqrt{v} x \sqrt{\Delta t})]
$$

where $x$ is a random draw from the unit normal distribution.

\section{CONSTANT ELASTICITY OF VARIANCE}

The CEV model ${ }^{5}$ is distinguished by its assumption that the underlying follows the stochastic process:

$$
\mathrm{d} S=\mu S \mathrm{~d} t+\sqrt{v} S^{\alpha / 2} \mathrm{~d} Z
$$

The discrete time version of (A5) is:

$$
S\left(t_{i+1}\right)=S\left(t_{i}\right)+\mu S\left(t_{i}\right) \Delta t+\sqrt{v} S\left(t_{i}\right)^{\beta-2 / 2} \sqrt{\Delta t} x
$$

where $x$ is a random draw from the unit normal distribution.

\section{STOCHASTIC VOLATILITY}

Heston $^{8}$ (see also Knoch, 1992) specifies geometric Brownian motion for the underlying and a square root process with a mean reversion term for the volatility parameter as follows:

$$
\begin{array}{r}
\mathrm{d} S=\mu S \mathrm{~d} t+\sqrt{v} S \mathrm{~d} Z_{1} \\
\mathrm{~d} v=\kappa[\theta-v] \mathrm{d} t+\sigma \sqrt{v} \mathrm{~d} Z_{2}
\end{array}
$$

Here, $\mu, \kappa, \theta$, and $\sigma$ are constants and $\mathrm{d} Z_{1}$ and $\mathrm{d} Z_{2}$ are standard Wiener processes whose increments have instantaneous correlation $\rho$. The discrete time versions of (A7) and (A8) are:

$$
\begin{aligned}
S\left(t_{i+1}\right) & =S\left(t_{i}\right) \exp \left[\left(\mu-v\left(t_{i}\right) / 2\right) \Delta t\right. \\
& \left.+\left(\sqrt{v\left(t_{i}\right)} x_{1} \sqrt{\Delta t}\right)\right]
\end{aligned}
$$


and,

$$
\begin{aligned}
v\left(t_{i+1}\right) & =v\left(t_{i}\right)+\kappa\left(\theta-v\left(t_{i}\right)\right) \Delta t \\
& +\left(\sigma \sqrt{v\left(t_{i}\right)} x_{2} \sqrt{\Delta t}\right)
\end{aligned}
$$

where $x_{1}$ and $x_{2}$ are determined by a random draw from the bivariate normal distribution with mean zero, unit variance, and correlation coefficient $\rho$.

\section{JUMP-DIFFUSION}

Merton's ${ }^{7}$ model is based on geometric Brownian motion augmented by a Poisson process. He thinks of the Poisson distributed event as the arrival of important information about the stock that affects its price. He assumes arrivals are independently and identically distributed. The probability of an event occurring during an interval of time $h$, where $h$ can be arbitrarily small, can be described as follows:

$\operatorname{Pr}$ \{event does not occur in the time interval

$$
(t, t+h)\}=1-\lambda h+\mathrm{O}(h),
$$

$\operatorname{Pr}\{$ event occurs once in the time interval

$$
(t, t+h)\}=\lambda h+\mathrm{O}(h),
$$

$\operatorname{Pr}\{$ event occurs more than once in the time interval

$$
(t, t+h)\}=\mathrm{O}(h)
$$

where $\mathrm{O}(h)$ is the asymptotic order symbol defined by $\psi(h)=\mathrm{O}(h)$ if $\lim _{h \rightarrow 0}[\psi(h) / h]$ is equal to zero, and $\lambda$ is the mean number of arrivals per unit of time. If a Poisson event occurs, the effect on the price of the stock is $S Y$ where $Y$ is a drawing of a random variable $Y$ from a distribution where all $Y$ are greater than or equal to zero, and successive draws of the random variable $Y$ are independently and identically distributed. The stochastic differential equation for the price of the underlying asset that reflects this combined jump and diffusion process is:

$$
\mathrm{d} S=(\alpha-\lambda k) S \mathrm{~d} t+\sqrt{v} S \mathrm{~d} Z+S \mathrm{~d} q
$$

where, following Merton's ${ }^{7}$ notation, $\alpha$ is the instantaneous expected return on the stock, $v$ is the instantaneous variance of the return conditional on no jumps in stock price occurring, $\mathrm{d} Z$ is, as before, a standard Wiener process, $q(t)$ is the independent Poisson process described in (A11), $\mathrm{d} q$ and $\mathrm{d} Z$ are independent, $\kappa \equiv E[Y-1]$ where $Y-1$ is the random variable percentage change in the stock price if the Poisson event occurs, and $E$ is the expectation operator over the random variable $Y$.

Merton ${ }^{7}$ points out that a closed form solution exists if the random variable $Y$ has a log-normal distribution.

Let the variance of the logarithm of $Y$ equal $\beta^{2}$ and define $\gamma \equiv \log (1+\kappa)$. Define the random variable:

$$
X_{n} \equiv \prod_{n} Y_{n}
$$

with the condition that:

$$
X_{0} \equiv 1
$$

The variable $n$ is the number of Poisson jumps that occur during the life of the option. $X_{n}$ is distributed lognormally with $E\left[X_{n}\right]=\exp [n \gamma]$ and the variance of the logarithm of $X_{n}$ equal to $\beta^{2} n$. Merton ${ }^{7}$ defines:

$$
f_{n}(S, T-t)=C\left(S, T-t, K, \sigma_{n}^{2}, r_{n}\right)
$$

where

$$
\sigma_{n}^{2} \equiv v+\frac{n \beta^{2}}{(T-t)}
$$


and

$$
r_{n} \equiv r-\lambda \kappa+\frac{n \gamma}{(T-t)}
$$

$C(\cdot)$ is the Black-Scholes pricing formula with the five input variables identified in (A15). The option pricing expression that follows from the stochastic specification in (A12), unpriced jump risk, and lognormally distributed random variables $Y_{n}$ is:

$$
\begin{aligned}
& F(S, T-t) \\
& =\sum_{n=0}^{\infty} \frac{e^{-\lambda^{\prime}(T-t)}\left(\lambda^{\prime}(T-t)\right)^{n}}{n !} f_{n}(S, T-t)
\end{aligned}
$$

where

$$
\lambda^{\prime} \equiv \lambda(1+\kappa)
$$

The discrete time version of (A12) is:

$$
\begin{aligned}
S\left(t_{i+1}\right)= & S\left(t_{i}\right) X_{n}\left(\Delta t_{i}\right) \exp [(\mu-v / 2-\lambda \kappa) \Delta t \\
& \left.+\left(\sqrt{v} x_{1} \sqrt{\Delta t}\right)\right]
\end{aligned}
$$

In (A20) $X_{n}\left(\Delta t_{i}\right)$ is the product of all of the $n$ jumps $Y_{n}$ that occur within the $i$ th time interval $\Delta t_{i} . X_{n}\left(\Delta t_{i}\right)$ is determined by first determining the number of jumps, $n$, in the time interval $\Delta t_{i}$ based on a random draw from the Poisson distribution with jump intensity parameter $\lambda \Delta t$. The size of each of the $n$ jumps is determined by making $n$ independent random draws from the distribution of $Y$. If there are no jumps in $\Delta t_{i}$, then $X_{n}\left(\Delta t_{i}\right)=X_{0}\left(\Delta t_{i}\right)=1$, as in (A4). If one jump occurs in $\Delta t_{i}, X_{n}\left(\Delta t_{i}\right)=X_{1}\left(\Delta t_{i}\right)=Y_{1}$. If more than one jump occurs in $\Delta t_{i}$, that is $n>1$, $X_{n}\left(\Delta t_{i}\right)$ is computed according to (A13).

The random variable $Y$ in the version of the jump-diffusion model which leads to the pricing expression in (A18) is distributed lognormally with $E[Y]=1+k$, and $\operatorname{VAR}[\ln (Y)]=\beta^{2}$. This is the model that is implemented in the simulations discussed in the body of the paper. In the simulations, draws are made from the distribution of $Y$ by first generating a random variable $x_{2}$ where $x_{2} \sim N(0,1)$ and defining:

$$
x_{2}^{\prime} \equiv \beta x_{2}+\ln (1+k)-\frac{\beta^{2}}{2}
$$

$Y$ is defined in the following way:

$$
Y \equiv e^{x_{2}^{\prime}}
$$

The transformations in (A21) and (A22) ensure that $x_{2}^{\prime} \sim N\left(\ln \left(1+k-\left(\beta^{2} / 2\right), \beta^{2}\right)\right.$ and $Y$ is distributed lognormally with mean $1+k$. 